\title{
Hand Writing recognition System using Neural Networks
}

\author{
M.Sujatha, V.Sandeep, Ch. Aishwarya, B.Mounika
}

\begin{abstract}
Handwritten Recognition is a process of pattern recognition which defines ability of a system to identify characters. There are many applications of Handwritten recognition (HWR) system such as reading postal addresses, bank check amounts, mail sorting and many more. HWR systems transcribes human written text into digital text. Plenty of research done in the field of recognizing handwritten characters but lacking in best accuracy is a challenge. In this proposed technique, offline HWR is done using Neural networks(NN) and Tensorflow is proposed. The proposed technique used to build a system which will be able to recognize the hand written characters with highest accuracy. The experiment is performed on proposed technique with accuracy of $85.5 \%$ compared to the state-of-the-art.
\end{abstract}

Indexed terms- Hand Written Recognition, Neural networks, Tensor flow.

\section{INTRODUCTION}

Hand Writing Recognition identifies the characters or words written by a user handwriting and converts into a format that the system understands. Most of the hand writing recognizers are now using Neural networks and Tensorflow. Neural networks performs a initial analyzing and categorizing the hand written input.Neural network must be trained repetitively by providing samples of hand written words as input and given feedback as to whether the Neural network guesses correctly or not at the interpretation of handwritten input. Neural networks consists of 3 layers namely Input layers, Hidden layers and Output layer.Tensorflow is a open-source software library.It is used for applications in ML such as Neural networks.It is symbolic math library which is used for both research and production. HWR is divided into two types, as offline and online. In online HWR method the 2D coordinates of successive points are represented as a function of time, whereas in offline HWR method the writing is usually captured optically by a scanner and is stored as an image.Offline HWR method is more familiar than online HWR because of the temporal information available within the former. Many applications like bank processing, document reading and postal address recognition require offline HWR system.

In this paper, a offline HWR[3] system using neural networks and tensorflow is proposed aiming to provide best accuracy.

Revised Manuscript Received on November 08, 2019.

Dr. M.Sujatha, Associate Professor, Jyothismathi Institute of Technology and Science, Ramakrishna Colony, Telangana

V.Sandeep, B.Tech Students Jyothismathi Institute of Technology and Science, Ramakrishna Colony, Telangana

Ch. Aishwarya, B.Tech Students Jyothismathi Institute of Technology and Science, Ramakrishna Colony, Telangana

B.Mounika, B.Tech Students Jyothismathi Institute of Technology and Science, Ramakrishna Colony, Telangana

\section{RELATED WORK}

1. J. Pradeep et al. [1] recommend that feature extraction and pre-processing techniques are applied to Input dataset, before the actual process is done. In their model (Neural Network) total number of Input units is the 54/69 and hidden units are 100 and output nodes are (26 alphabets, 0-9 Numerals) and they will use the sigmoid function in order to collect the weights of each unit.

2. N. Sharma et al. [2] explained classifier techniques give the accuracy results in achieving the Handwritten words (include characters, digits). They proposed a model which trained with digits and characters, but they use a smaller number of hidden units compared to the other model. But in their model, it will not recognize the handwritten characters properly, because sometimes error rate will occur, without hidden units it is difficult to find the perceptron which raise the error. But their model is very good if the Inputs (characters, digits) are correctly trained to the model.

\section{METHODOLOGY}

Neural Networks are organized within layers. Neural Network consists of 3 layers. Patterns are defined to network using input layer, which further communicates with hidden layers where actual processing is done. The hidden layers then links to output layer where output is stored. Neural Networks are not sequential or deterministic. There is no separate memory addresses for storing data in Neural Networks. The network is trained using a sample data set and appropriate using algorithm for further estimation.[4] Key advantages of neural networks are they have an ability to learn non-linear data, it makes the data generalized and predict on[6] unseen data. Lack of interpretability and need for a strong signal in data are some of the limitations of Neural Networks.

\section{A. Problem Definition:}

In the problem definition we are described that how to recognize the characters which includes both alphabets and digits.In this kind of problem we are basically using the approach of classification i.e., Neural Network. In this paper we are recommending that we made a one NN plays a great role in predictions other than all classification algorithms So we can adopt NN as classification model for given problem.

\section{B. Proposed Technique:}

In this section, the proposed recognition is described. First, we will give the dataset (Input) which contains the large number of data tuples (digits, characters). If the data is noise or any other irrelevant, we need to pre-process the data by using pre-processing techniques. We have built Neural Network that contains 36 Input units and 1000 Hidden units for each Input unit and we have 36 output units (0-9 digits,26 Alphabets)." Input is divided into some pixels

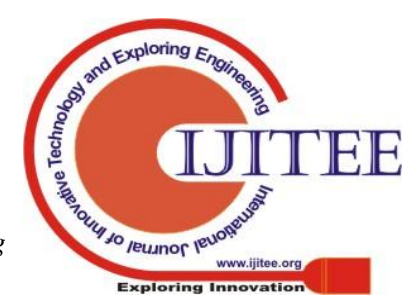




\section{Hand Writing recognition System using Neural Networks}

according to the size used in the sample dataset.In order to get different digits and alphabets we changed test value in code executed."

These kinds of models Neural Networks are already available, but our model is also similar to other Neural Networks model for recognizing the Handwritten characters. But we do that increasing the weight of each Input Node, and units automatically decreases the error rates[5] (sample error) and we much paid attention to the increasing the Hidden units, so that Back propagation is easy process. The following diagrams shows our model:
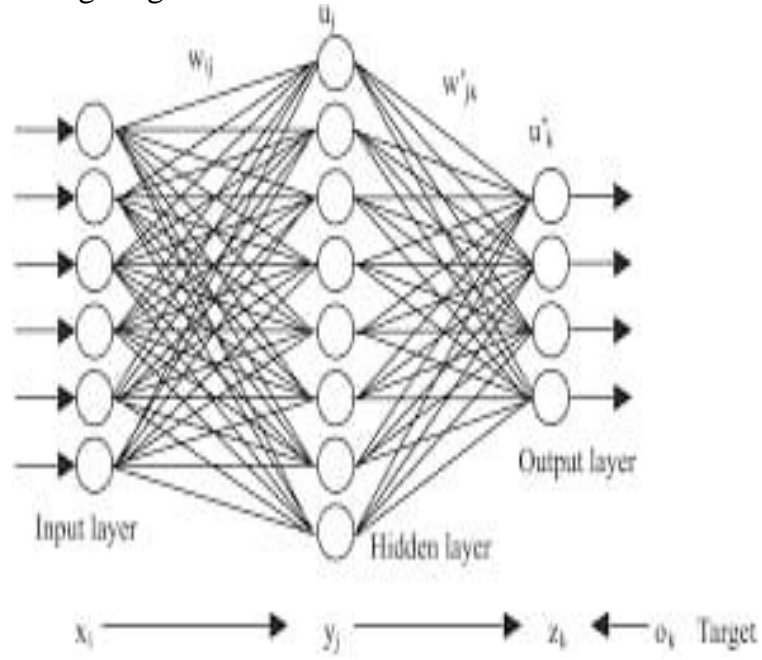

Figure1:Overview of Neural network layers in predicting the characters

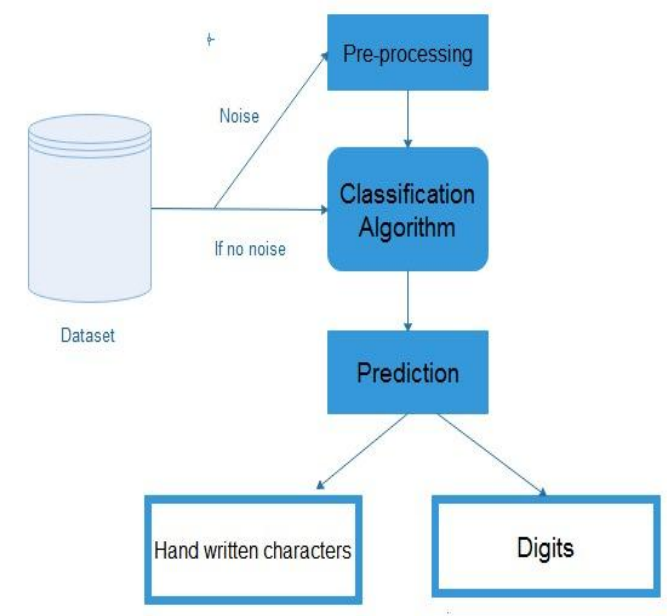

Figure2 : System Architecture

The above figure shows the system architecture of our proposed model.In which firstly, dataset will be provided to the model.If the dataset contain noise preprocessing techniques will be applied,otherwise directly given to the model so that it can predict the characters.

\section{EXPERIMENTAL ANALYSIS AND RESULTS}

In the proposed HWR system Neural network architecture was used and trained with a dataset. The main aim of this system is to predict the hand written characters in digital text format, predictions of some characters and digits are shown in figures with an accuracy of $85.55 \%$.

[2]

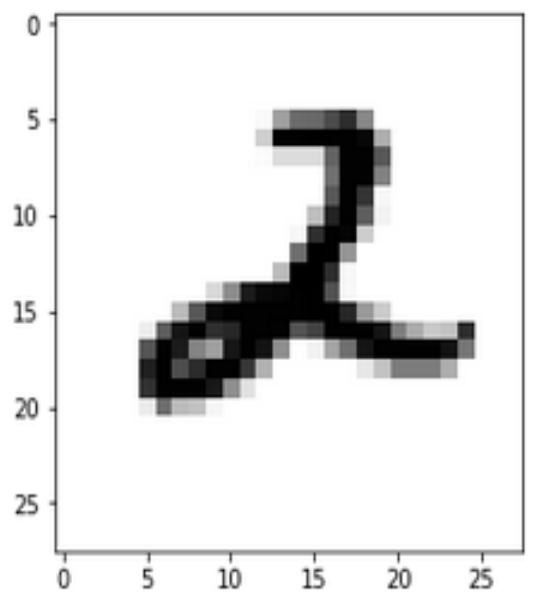

Figure3:Prediction of $\mathrm{HW}$ character of digit 2 as digital text.

[8]

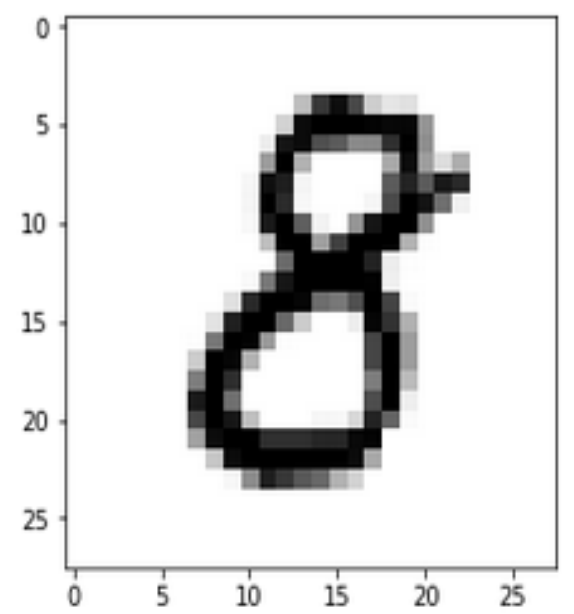

Figure4:Prediction of $\mathrm{HW}$ character of digit 8 as digital text.

[3]

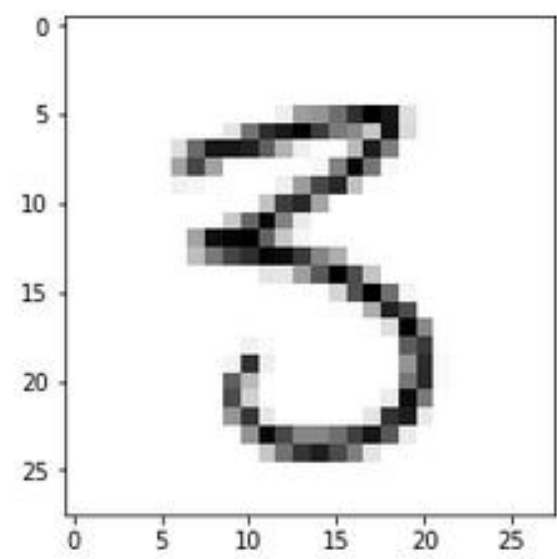

Figure5:Prediction of $\mathrm{HW}$ character of digit 3 as digital text. 
[0]

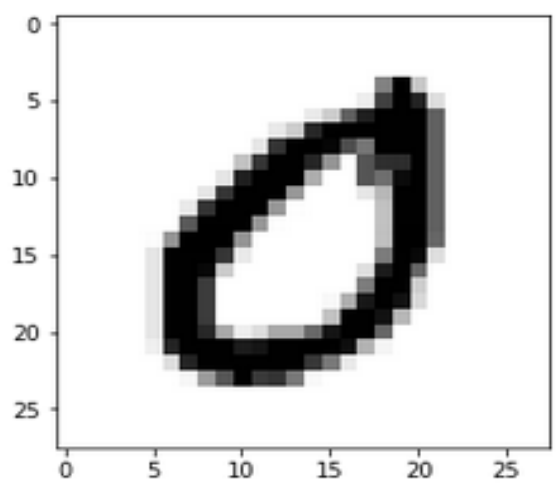

Figure6:Prediction of $\mathrm{HW}$ character of digit 0 as digital [1]

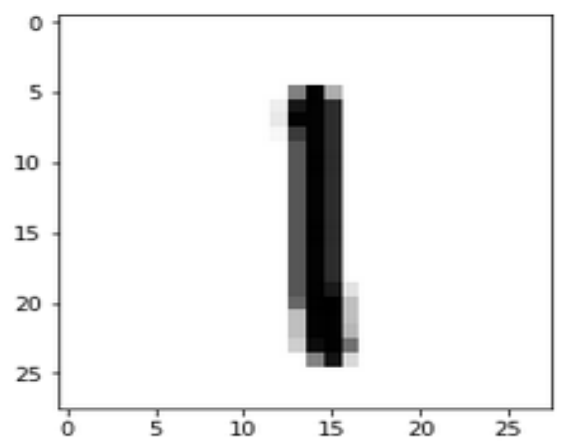

Figure7:Prediction of $\mathrm{HW}$ character of digit 1 as digital text.

[9]

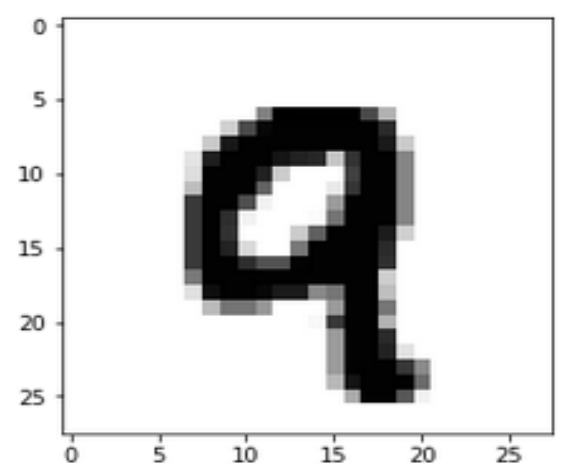

Figure8: Prediction of $\mathrm{HW}$ character of digit 9 as digital text. [6]

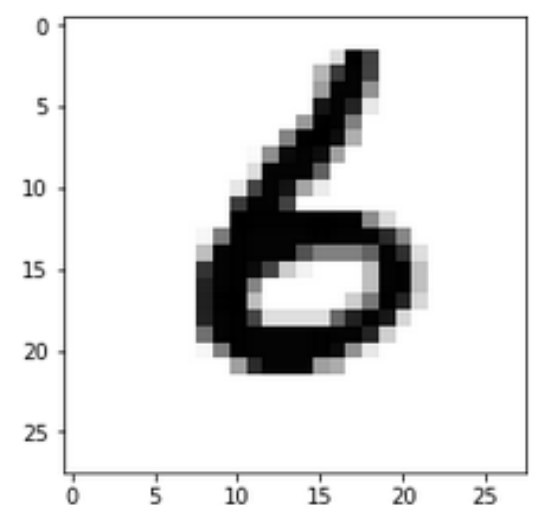

Figure9:Prediction of HW character of digit 6 as digital text.
[4]

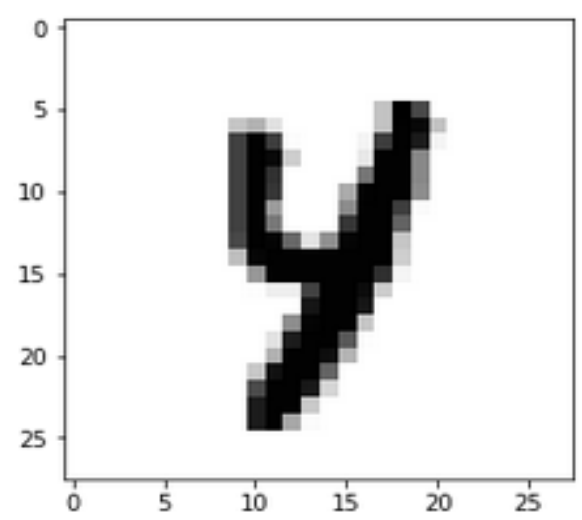

Figure10:Prediction of HW character of digit 4 as digital text.

[5]

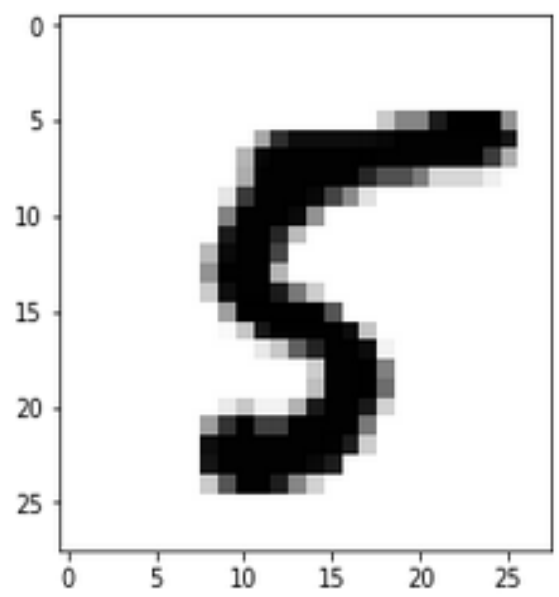

Figure11: Prediction of $\mathrm{HW}$ character of digit 5 as digital text.

[7]

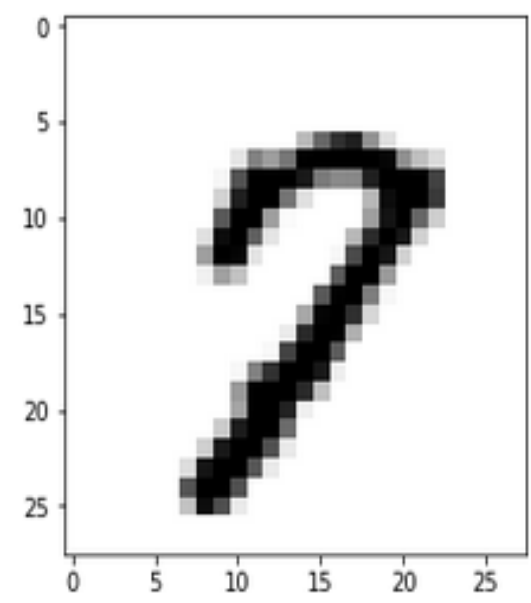

Figure12: Prediction of $\mathrm{HW}$ character of digit 7 as digital text. [B] 


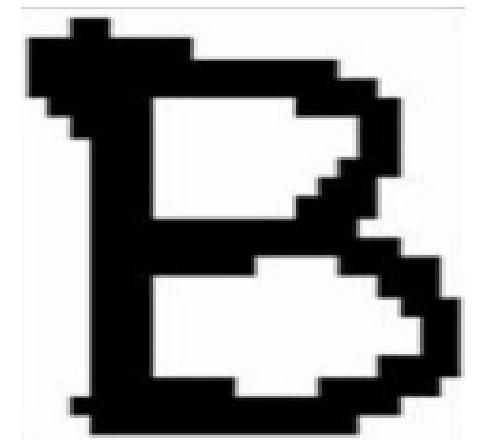

Figure13: Prediction of HW character of alphabet B as digital text

[I]

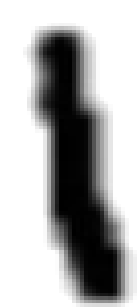

Figure14:Prediction of HW character of alphabet $I$ as digital text.

\section{CONCLUSION}

Finally, we conclude that a simple model HWR is constructed using neural networks and tensor flow in proposed technique.Hand written recognition helps to recognize the patterns of the digits which was mentioned in dataset. According to the model,after training the dataset,testing will done.To compare the recognition efficiency of the proposed system, the neural network system is trained using a sample dataset. Proposed offline HWR helps in converting of any Hand written character into structured text format.

\section{REFERENCES}

1. J.Pradeep, E.Srinivasan and S.Himavathi,'Diagonal Based Feature Extraction For Handwritten Alphabets Recognition System Using Neural Network",International Journal of Computer Science \& Information Technology (IJCSIT), Vol 3, No 1, Feb 2018 Pondicherry, India.

2. N.Sharma, U.Pal, F.Kimura, "Recognition of Handwritten digits",9th International Conference on Information Technology(ICIT06),2017.

3. Megha Agarwal, Shalika, Vinam Tomar, Priyanka Gupta,Handwritten "Character Recognition using Neural Network and Tensor Flow",International Journal of Innovative Technology and Exploring Engineering (IJITEE),ISSN: 2278-3075, Volume-8, Issue- 6S4, April 2019

4. Akm Ashiquzzaman and Abdul Kawsar Tushar,"Handwritten Arabic Numeral Recognition using Deep Learning Neural Networks",University of Asia Pacifific, Dhaka, Bangladesh.

5. N. Arica and F. Yarman-Vural, "An Overview of Character Recognition Focused on Off-line Handwriting", IEEE Transactions on Systems, Man, and Cybernetics, Part C: ,Applications and Reviews, 2016, 31(2)

6. K.GauravandBhatiaP.K.,"Analytical Review of Preprocessing techniques for Offline Handwrittten Character Recognition”, 2nd International Conference on Emerging Trends in Enginerring \& Management,ICETEM, 2019.

\section{AUTHORS PROFILE}

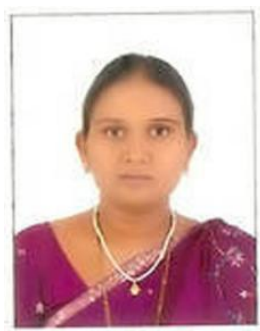

Author 1: Dr.MADUGULA SUJATHA, Working as Associate Professor in CSE at Jyothishmathi Engineering college, Karimnagar. She pursued Ph.D from Andhra University in 2017. She has 12 years teaching experience in various engineering colleges She is expert in Data mining, Machine learning domains etc.

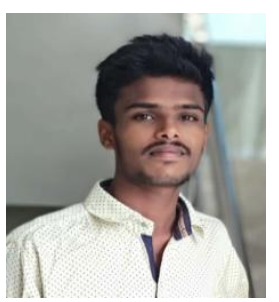

Author 2: V.Sandeep, is pursuing B.Tech (Computer Science and Engineering) in Jyothishmathi Institute of Technology and Science in Karimnagar, India.

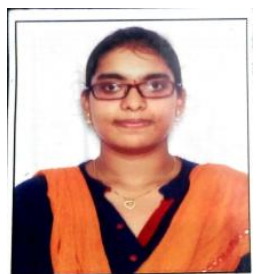

Author 3: Ch.Aishwarya, is pursuing B.Tech (Computer Science and Engineering) in Jyothishmathi Institute of Technology and Science in Karimnagar, India.

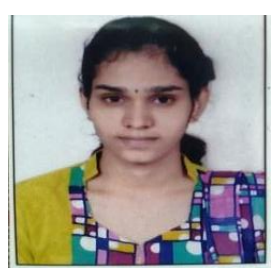

Author 4: B.Mounika, is pursuing Computer Science and Engineering in Jyothishmathi Institute of Technology and Science in Karimnagar, India. 